\title{
APPLICATION OF TEM ANALYSIS IN THE INTERPRETATION OF THERMAL DESORPTION SPECTRA OF NITROGEN ADSORBED ON IRON FILMS
}

\author{
Enrico. G. Keim ${ }^{*}$ and Wojtek Lisowski** \\ ${ }^{*} \mathrm{MESA}^{+}$Research Institute, Central Materials Analysis Laboratory, University of Twente, P.O. Box \\ 217, 7500 AE Enschede, The Netherlands \\ ${ }^{* *}$ Institute of Physical Chemistry, Polish Academy of Sciences, Kasprzaka 44/52, PL-01-224 \\ Warszawa, Poland
}

High temperature adsorption of nitrogen on iron was intensively studied. It is a crucial step in the ammonia synthesis [1]. Thermal desorption mass spectrometry (TDMS) is a widely applied experimental method which allows the nitrogen surface adsorption states, appearing at various nitrogen pressures and temperatures, to be distinguished and characterized [2]. However, an interpretation of the TDMS results is complicated by the structural transformation within the iron films as a result of both nitrogen adsorption at high temperature and TDMS heating process. In this work it is our aim to present experimental data that deal with the question of how the bulk structure of iron films is affected by nitrogen induced transformation in their topmost surface region at elevated temperatures and how the bulk transformation of iron films affects the TDMS spectra. For this purpose we used the combination of TDMS and Transmission Electron Microscopy (TEM).

The TDMS experiments as well as the evaporation of Fe samples for TEM analysis were performed in a glass UHV apparatus [3]. Each time thin Fe films were deposited under UHV conditions onto a clean, quartz substrate $(10 \times 10 \mathrm{~mm}$ in size and $1.5 \mathrm{~mm}$ thick), placed within the quartz TDMS cell. After evaporation the film samples were annealed "in situ", at a vacuum pressure lower than $10{ }^{-6} \mathrm{~Pa}$ [4]. The TDMS procedure was subsequently repeated "in situ" each time for a new sample, until the most interesting stage of the TDMS process was reached. Consequently, we were able to prepare specimens representative of the Fe films after successive steps of the TDMS process, i.e., after annealing at $700 \mathrm{~K}$, after nitrogen adsorption at $673 \mathrm{~K}$ and after thermal desorption, respectively. Morphological and structural examination of the iron films was performed "ex situ" using TEM (Philips CM30 Twin (S)TEM). The TEM specimens were prepared in cross-section using dimpling and dual beam ion etching [4].

Four thermal desorption states can be distinguished in the complex TDMS spectrum (Fig. 1). The states A, B and C can be interpreted as a superposition of various surface nitrogen desorption states. State D originates from nitrogen released from intergranular spaces of the iron nitride film as a result of its high-temperature reconstruction. This conclusion is deduced from an analysis of the crosssectional TEM images of Fe films taken after successive steps in the TDMS process (Fig 2). The nitrogen-induced reconstruction of the surface and subsurface region of the iron film involves also its bulk structure rearrangement although no nitrogen is detected within this region. Mainly the family of the low-index planes, e.g., [100]bcc, [210]bcc and [211]bcc (not shown here), attributed to iron nitrides $[5,6]$, was observed. The recrystallization process of the Fe film, which is activated during the TDMS heating to $950 \mathrm{~K}$, is accompanied by an increase in the mean surface grain size 
(Fig. 2(c)) and a distinct ordering of the crystallite structure. As a result we obtain a film which is similar to a pure iron film annealed to $1000 \mathrm{~K}$ [4].

\section{References}

[1] M. Grunze, in "The Chemical Physics of Solid Surfaces and Heterogeneous Catalysis" vol. 4, Eds. D.A. King and D.P. Woodruff, (Elsevier Scientific Publishing Company, Amsterdam, 1982) p. 143.

[2] D.A. King, Surf. Sci. 47, 384 (1975).

[3] W. Lisowski, Vacuum 53, 13 (1999).

[4 ]W. Lisowski, E.G. Keim, M.A. Smithers, Appl. Surf. Sci. 189, 148 (2002).

[5] Y. Takahashi, M. Katou, H. Shoji, M. Takahashi, J. Magn. Magn. Mater. 232, 18 (2001).

[6] N.D. Telling, G.A. Jones, C.A. Faunce, P.J. Grundy, H.J. Blythe, D.E. Joyce, J. Vac. Sci. Technol. A 19, 405 (2001).

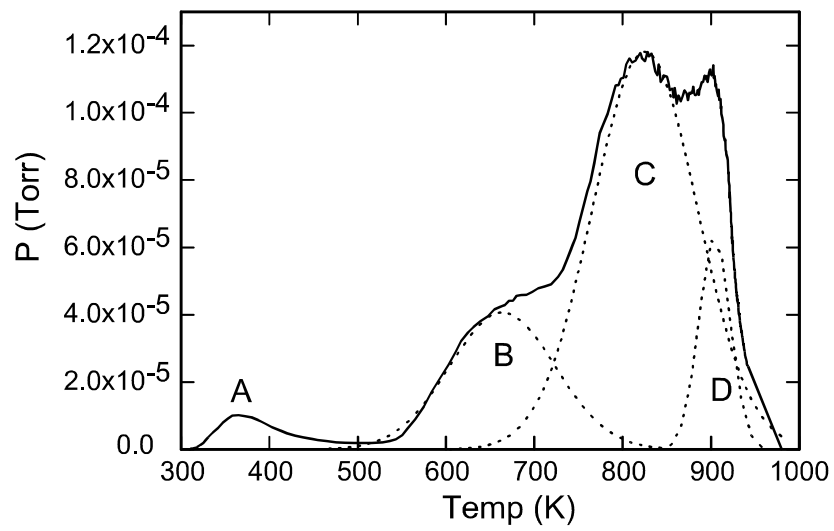

FIG. 1: TDMS spectra of nitrogen from a thin Fe film. The adsorption temperature was $673 \mathrm{~K}$ and the final equilibrium nitrogen pressure was $0.75 \mathrm{~Pa}$. Dotted lines indicate a Gaussian deconvolution of the complex part of the TDMS spectrum.

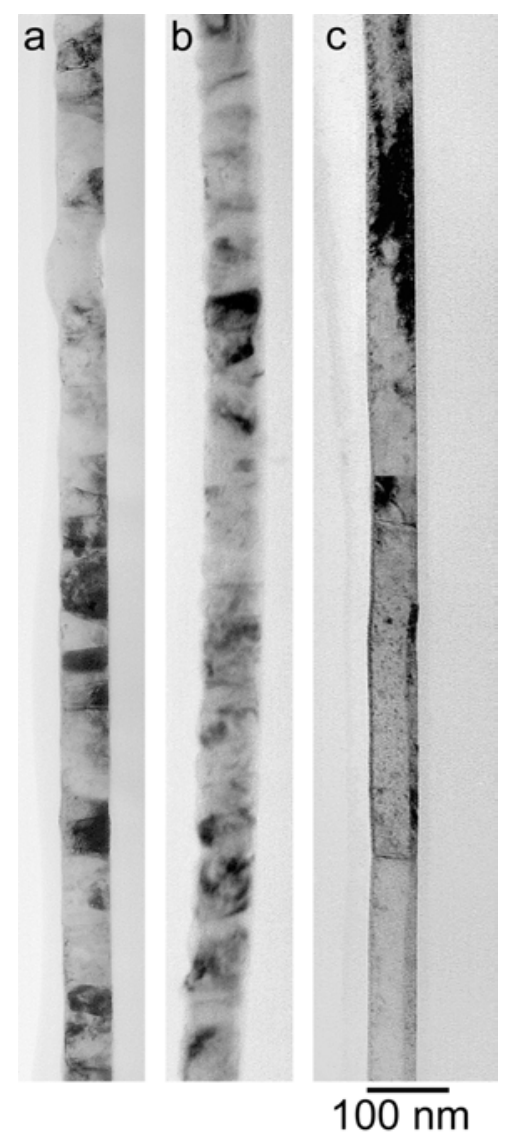

FIG. 2: Cross-sectional TEM images of: (a) the $\mathrm{Fe}$ film annealed in vacuum at $700 \mathrm{~K}$ for 60 min., (b) the Fe film surface in (a), after exposure to nitrogen $(0.75 \mathrm{~Pa})$ at $673 \mathrm{~K}$ and cooling to $300 \mathrm{~K}$, (c) the Fe film surface in (b), after the heating cycle in the TDMS process from $300 \mathrm{~K}$ up to $950 \mathrm{~K}$. The thickness of the films is: $54 \pm 2 \mathrm{~nm}$ (a), $68 \pm 6 \mathrm{~nm}$ (b), $55 \pm 2$ $\mathrm{nm}(\mathrm{c})$. 